\title{
Accuracy Estimation of the Black Sea Circulation Modeling Results Obtained at Different Bottom Topography
}

\author{
O. A. Dymova*, N. A. Miklashevskaya \\ Marine Hydrophysical Institute of RAS, Sevastopol, Russian Federation \\ * olgadym@yahoo.com
}

\begin{abstract}
Purpose. Accuracy of the reconstructed hydrophysical fields calculated using different data on bottom topography is estimated in order to determine the depth array corresponding to the modern tasks of the Black Sea circulation modeling with high spatial resolution.

Methods and Results. Two numerical experiments on modeling the circulation were carried out based on the Marine Hydrophysical Institute, Russian Academy of Sciences (MHI RAS) ocean model. Horizontal resolution was $1.6 \mathrm{~km}, 27$ irregular $z$-horizons were preset vertically and the SKIRON/Eta data (2011) were used as the atmospheric forcing for both cases. Difference between the experiments consisted in application of different bathymetry. In the first experiment, the bottom topography was preset in accordance with the Black Sea depths from the MHI Ocean Data Bank with the 5-minute resolution; in the second one - based on the European Marine Observation and Data Network (EMODnet) depth array with the $1 / 8^{\prime}$ resolution. The calculated hydrophysical fields were compared with the temperature and salinity measurements, and satellite images of the sea surface temperature. The analysis showed that application of the depths data of higher resolution permitted to improve accuracy of thermohydrodynamic characteristics of the Black Sea circulation in the 30-300 m layer. The integral values of the eddy kinetic energy and the mean current kinetic energy for two experiments were also considered for both of the experiments. The results of the comparative analysis demonstrate the fact that, at the bottom topography with higher resolution taken into account, in the simulated system the mechanisms of energy redistribution between currents and eddies changed during intensive storm impacts.

Conclusions. The results of the present research permit to conclude that in the experiment with a smoother bottom relief, increase of kinetic energy both of the eddies and currents was due to barotropic instability. In case of more complex bathymetry, the eddy kinetic energy increased mainly owing to the processes associated with baroclinic instability.
\end{abstract}

Keywords: Black Sea, modeling, bathymetry, EMODnet, in situ data, current, eddy, kinetic energy.

Acknowledgements: the authors are grateful to the reviewers for their helpful comments. Experiment 1 and comparative analyses were performed in the framework of the state task № 08272019-0003 "Fundamental study of oceanological processes conditioning state and evolution of marine environment under effect of anthropogenic factors based on observational and modeling methods". The EMODnet data adaptation for the MHI-model and experiment 2 were carried out under support of the Russian Foundation for Basic Research (project No. 18-05-00353 A).

For citation: Dymova, O.A. and Miklashevskaya, N.A., 2019. Accuracy Estimation of the Black Sea Circulation Modeling Results Obtained at Different Bottom Topography. Physical Oceanography, [ejournal] 26(4), pp. 304-315. doi:10.22449/1573-160X-2019-4-304-315

DOI: $10.22449 / 1573-160 \mathrm{X}-2019-4-304-315$

(C) 2019, O. A. Dymova, N. A. Miklashevskaya

(C) 2019, Physical Oceanography

\section{Introduction}

The real picture reconstruction of physical, biological, chemical and other processes occurring in the coastal zones of marine waters is an urgent task of modern oceanology. Numerous systems for observing, analyzing and forecasting changes in the marine environment are deployed in the vicinity of the shores of 
the seas and oceans in order to ensure both the hydrosphere's resource potential preservation and the protection of national economy objects from the devastating consequences of natural phenomena occurring in the ocean. One of the main ways for obtaining information about the past, present and possible future state of water systems is numerical modeling. At the same time, the quality of diagnosis and forecast depends on the correct consideration of numerous factors, among which input data describing atmospheric effects, river runoffs, bathymetry and coast line orography play an important role. A characteristic feature of the Black Sea bottom relief is the shelf narrowness and a steep continental slope (slope reaches 20-30 in the northeastern and southern parts of the sea. The Black Sea is one of the few basins with such a steep continental slope. Therefore, bottom topography is essential for modeling dynamics. This work is devoted to the analysis of the effect of bottom topography features on the Black Sea circulation structure.

In recent decades Marine Hydrophysical Institute (MHI) of RAS has been actively working on numerical modeling of the Black Sea thermo-hydrodynamic regime [1, 2] on the basis of MHI model [3], which uses the basin bathymetry taken from the MHI Ocean Data Bank (MHI ODB) [4]. Initially, this array of depths was constructed for circulation calculations with about $15 \mathrm{~km}$ spatial resolution [5]. Subsequently, with an increase in the MHI model resolution [1, 6] the array was interpolated to a finer grid. In this approach, important features of coastal zones are not taken into account at the grid nodes located close to the coast. This introduces inaccuracy in the modeling results [7]. Therefore, the refinement of data on the sea depths for more accurate description of hydrophysical fields seems to be an urgent task.

As an alternative, we chose the Black Sea bathymetry, provided for free by the European Marine Observation and Data Network EMODnet (http://portal.emodnet-bathymetry.eu/). This array has 1/8' resolution (about $200 \mathrm{~m}$ ) and is based on a digital relief model that is generated from bathymetric surveys, satellite information and GEBCO data. Today this is the maximum spatial resolution for data covering the entire sea, which are publicly available. It is shown in [8] that the product has a high degree of feasibility indicator (more than $70 \%$ ) and is recommended for use. The depth maps $(h)$ constructed according to MHI ODB and EMODnet are shown. It can be seen that EMODnet data reconstruct such characteristic features of the sea bottom as a paleochannel of the Danube, the heterogeneity of the relief in the western and eastern sea corners and fine features of continental slope in the north-western shelf region and the Caucasus coast. The sea zone contoured by $2000 \mathrm{~m}$ isoline, occupies in Fig. 1, $b$ a larger area than in Fig. 1, $a$. Also the correspondence between the depth and the coastline isolines near the eastern and western basin boundaries is noticeably higher. Visual analysis showed that the depth change on the map, created on the basis of EMODnet materials, corresponds to the official data of bathymetric maps approved by the Federal Agency for Geodesy and Cartography of Russia [9].

The calculations for modeling the Black Sea circulation with bathymetry obtained from the MHI ODB and EMODnet were carried out in this work. The results are compared with the data of contact and satellite observations. Based on the results of numerical experiments, the estimates of eddy and current kinetic energy change over the time depending on the depth array used are made. 


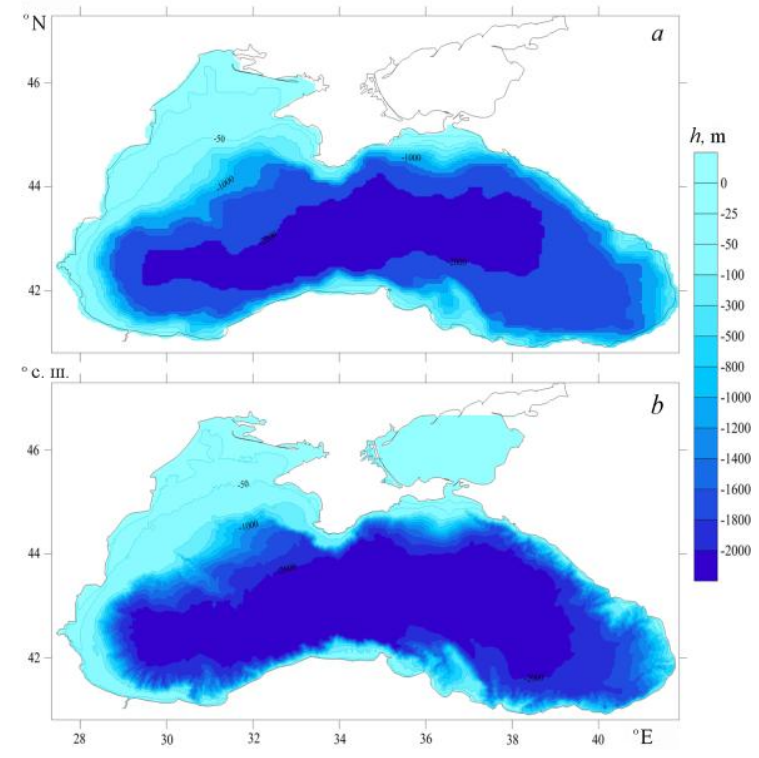

F i g. 1. Charts of the Black Sea depths constructed based on the MHI Ocean Data Bank (a) and EMODnet $(b)$

\section{Statement of the problem}

Numerical modeling was carried out using the eddy-resolving energy-balanced MHI model [3]. The model is constructed on the basis of ocean thermohydrodynamics primitive equations, written down in a Cartesian coordinate system, in the Boussinesq approximation, hydrostatics and incompressibility of sea water. We neglect the compressibility and, consequently, the dependence of density on pressure, since the depth in the Black Sea is much shallower than in the ocean.

In the MHI model, the density non-linearly depends only on temperature and salinity and is calculated by O.I. Mamaev formula [10]. The level field was calculated using the linearized kinematic condition on the surface, which allows one to study fast unsteady processes that play important in coastal zones [11]. Heat fluxes, precipitation, evaporation, and wind stress were specified as boundary conditions on the sea surface. Also, the assimilation of sea surface temperature (SST) was included in the model. The assimilation procedure consisted in calculating the temperature discrepancy with the help of which the model temperature was corrected then. Further, in order to filter out small-scale perturbations, the diffusion equation was solved for the corrected surface temperature. The assimilation procedure, the form of equations and boundary conditions for them are presented in detail in [3]. Correction of calculated temperature and salinity by depth using climatic profiles was not carried out. Vertical turbulent mixing is parameterized using the Mellor - Yamada turbulent closure model [12]. The coefficients of horizontal turbulent viscosity and diffusion are constant. At the bottom the conditions of impermeability and the absence of heat and salt fluxes were set, friction on the bottom was not taken into account. Slip condition and impermeability conditions were fulfilled on rigid lateral sections of the boundary; normal temperature derivatives were set to zero for temperature 
and salinity. The temperature, salinity and velocity of currents in the estuaries and straits corresponded to climatic data [13]. At the initial time, the level elevation, temperature, salinity and horizontal current velocities were set. MHI model is implemented on a grid $C$ [14] with $1.6 \mathrm{~km}$ step along spatial coordinates. 27 nonuniform $z$-horizons were selected vertically. $x$ axis is directed to the east, $y$ axis - to the north and $z$ axis - vertically down. Usually ocean models use potential temperature as a predictor variable. As the Black Sea depth is about two kilometers, it practically does not matter whether the potential temperature or in situ temperature is taken as an argument (the error does not exceed $0.001{ }^{\circ} \mathrm{C}$ [10]). In MHI model adiabatic correction is not taken into account, and further by the term "temperature" we mean in situ temperature. A complete description of the model equations and boundary conditions was performed in [3].

Two numerical experiments were carried out. In experiment 1 , an array of depths was taken from MHI ODB. Experiment 2 was performed using EMODnet data, smoothing filters were used to construct new depth array. Atmospheric forcing in two experiments was set according to the fields obtained by SKIRON/Eta model (http://forecast.uoa.gr/forecastnewinfo.php) for 2011 with $1 / 10^{\circ}$ horizontal resolution in latitude and longitude. Sea surface temperature is also taken according to SKIRON/Eta data. As the initial fields we used the level, three-dimensional temperature, salinity and horizontal velocities of currents for January 1 of the climatic year, obtained from the climate calculation using MHI model on a $5 \mathrm{~km}$ grid with bottom topography from MHI ODB [15]. Prior to the calculations, the depth arrays and boundary fields were linearly interpolated to the model grid nodes. Initially, the depth arrays are different, so the number of boxes filled with liquid is not the same in the experiments. Previously, an increase in the spatial resolution of $\mathrm{MHI}$ model was realized inside the old computational domain (identical to the basin in [15]). Therefore, the initial field for experiment 1 was constructed by linear interpolation of the climatic fields to the nodes of the new grid. Further, this field was checked for compliance with EMODnet bathymetry: in the absence of hydrodynamic data in the cell, the fields were extrapolated horizontally from the nearest non-zero values. When reaching land or continental slope, the cells were marked as land/bottom. The new array was smoothed, checked for compliance with the boundary conditions, and then was used as the initial field for experiment 2 .

In order to coordinate the initial and atmospheric fields in each experiment, a quick quasi-geostrophic adjustment procedure was performed: during the first four days the model equations were integrated under a fixed atmospheric effect corresponded to January 1, 2011. The obtained arrays of thermo-hydrodynamic characteristics were used as starting points. Both experiments were performed for one year period. The fields of sea level, temperature, salinity and velocity components were averaged over one day, and these arrays were recorded as output data for each day.

\section{Comparison with observational data}

To assess the accuracy of the simulation results, we compared the hydrophysical fields with the data of available contact observations in accordance with the fourth class of MERSEA diagnostics [16]. Model temperature 
and salinity were compared with measurements of three Argo buoys (1901200, 6900803, 7900466), which profiled to $1500 \mathrm{~m}$ depths (http://usgodae.org/argo/argo.html). We considered 192 profiles. For each profile Argo and modeling data are reduced to 6 characteristic layers at depths corresponding to the vertical levels of the model, and the residuals between the measured and model values at a certain point are calculated. Further, the rootmean-square deviations (RMS) of the temperature and salinity residuals at characteristic horizons were found for all profiles. The results obtained in the first and second experiments are given in the table. Hereinafter, subscripts 1 and 2 correspond to the number of the experiment. For both calculations the maximum values of RMS of the residuals were revealed in 5-30 m layer for temperature and in 30-100 m layer for salinity. It can be seen that the maximum values of $\mathrm{RMS}_{2}$ of residuals of both temperature and salinity decrease in these layers, however, an increase in $\mathrm{RMS}_{2}$ of residuals of temperature in 0-5 m layer and salinity in 5-30 m layer is observed. At 100-300 m horizons for temperature and salinity residuals $\mathrm{RMS}_{2}$ is smaller than $\mathrm{RMS}_{1}$. Below $300 \mathrm{~m}$, the data of experiments 1 and 2 insignificantly differ from each other: in 300-800 m layer $\mathrm{RMS}_{2}$ of the residuals of both parameters slightly increases, in 800-1500 m layer it decreases.

Root-mean-square deviations between the measured and calculated temperature and salinity in experiments 1 and 2

\begin{tabular}{c|c|c|c|c}
\hline \multirow{2}{*}{ Depth, m } & \multicolumn{2}{|c|}{ Temperature } & \multicolumn{2}{c}{ Salinity } \\
\cline { 2 - 5 } & $\mathrm{RMS}_{1}$ & $\mathrm{RMS}_{2}$ & $\mathrm{RMS}_{1}$ & $\mathrm{RMS}_{2}$ \\
\hline $0-5$ & 0.182 & 0.239 & 0.054 & 0.050 \\
$5-30$ & 0.673 & 0.507 & 0.035 & 0.043 \\
$30-100$ & 0.212 & 0.203 & 0.481 & 0.424 \\
$100-300$ & 0.049 & 0.035 & 0.111 & 0.058 \\
$300-800$ & 0.004 & 0.006 & 0.041 & 0.048 \\
$800-1500$ & 0.011 & 0.003 & 0.011 & 0.0005 \\
\hline
\end{tabular}

\section{Analysis of current velocity fields}

Comparison of the results of two numerical experiments revealed the following differences between them. In the calculation with topographic data of higher resolution, the Rim Current velocity (RC) decreased. If average velocity was $30-50 \mathrm{~cm} / \mathrm{s}$ when using MHI ODB depth array, then it varied in $15-35 \mathrm{~cm} / \mathrm{s}$ range when using EMODnet data. In addition, according to the results of experiment 2, in summer period the Rim Current meandering increased in the areas of Sevastopol and Batumi anticyclones formation, as well as along the Caucasus coast. In experiment 2, in the region of the Crimean and Anatolian coasts, the formation of a larger number of mesoscale eddies than in experiment 1 was observed.

The pattern of surface currents in both experiments was significantly different at the stage of Batumi anticyclone formation. In experiment 1, in late March - early April at around $40{ }^{\circ} \mathrm{E}$ a chain of two anticyclones was observed. It intensified and merged into one by the end of April. In late May, a cyclonic eddy was formed to 308

PHYSICAL OCEANOGRAPHY VOL. 26 ISS. 4 (2019) 
the southwest of the anticyclonic gyre. By early June, it increased in size and existed until early July. In experiment 2, as a result of the Rim Current meandering in the abyssal zone of the southeastern sea part, an eddy dipole was formed. Batumi anticyclone later emerged from it in consequence of the strengthening of dipole anticyclonic part due to influence of mesoscale eddies of the Anatolian coast and attenuation of the dipole cyclonic part. Formation of a coastal cyclone to the southwest from Batumi eddy was not recorded in experiment 2.

In experiment 1 in late June, a cyclonic gyre began to form northward from Batumi anticyclone (inside the Rim Current). In experiment 2, such an eddy formed a month later, in late July. In experiment 1 , by the end of September the cyclone significantly exceeded the size of the anticyclone but its velocity was lower than in the anticyclone: $5-20 \mathrm{~cm} / \mathrm{s}$ versus $30-50 \mathrm{~cm} / \mathrm{s}$ in the anticyclone. In experiment 2 the cyclone also intensified, but its size did not exceed the anticyclone diameter: in the zonal direction it was much narrower than the anticyclone. In both experiments the Batumi anticyclone was enhanced by mesoscale eddies formed in the eastern part of the Anatolian coast. However, in experiment 2 the merge phase of the mesoscale eddies with the Batumi anticyclone was shifted in time by about a month. The anticyclone dissipation phase in experiment 2 was also observed a month later, in late November.

A difference in the phases of the eddy evolution was revealed for the Sevastopol anticyclone in two experiments. The total number of eddies observed in the Sevastopol anticyclone region in experiment 1 is greater than in the same zone according to experiment 2. Until the end of March, the current fields by the results of both calculations are qualitatively close. In experiment 2 , from mid-April the life time of the Sevastopol anticyclone increased in comparison with eddies from experiment 1 . Thus, in experiment 1 anticyclonic eddies occurring in the Crimean Peninsula region and moving along the Rim Current periphery to the southwest dissipated in the Bosphorus region in 3-4 months. In experiment 2 only two gyres were formed sequentially (with almost 4 months difference). The first one, occurred in April, was transported with the Rim Current jet to the western part of the Anatolian coast and was observed until the end of the computational period. The second eddy was formed at the end of August and also existed until the end of the computational period. It should be noted that the movement velocity of both gyres down the current slowed down in comparison with the results of experiment 1 .

In an experiment with bathymetry data from MHI ODB, it was found that as a result of the Rim Current meandering in early April, a long-lived anticyclonic gyre with $\sim 100 \mathrm{~km}$ diameter and $10-25 \mathrm{~cm} / \mathrm{s}$ characteristic velocity was formed in the eastern part of the sea. Its center was located in $42.5^{\circ} \mathrm{N}, 38.5^{\circ} \mathrm{E}$ area. By the end of November the anticyclone moved to the northwest and its center was already located in $43{ }^{\circ} \mathrm{N}, 36.5^{\circ} \mathrm{E}$, while the velocity increased to $30-40 \mathrm{~cm} / \mathrm{s}$. The mentioned eddy was clearly observed from the surface to $400 \mathrm{~m}$ horizon. In the lower layers the velocity decreased to $3-7 \mathrm{~cm} / \mathrm{s}$. In experiment 2 this anticyclone was not reconstructed.

In experiment 2 (EMODnet) several significant anticyclonic gyres, which were absent in the results of experiment 1, were observed. Since the end of April a small eddy of the anticyclonic vorticity sign (Sukhumi anticyclone) with 30-35 cm/s 
velocity was formed in the Sukhum region. By early July it had displaced half a degree northward in the direction of the Rim Current movement. During this period it noticeably increased in size, reaching $\sim 100 \mathrm{~km}$ in diameter. At the same time, the velocity in the anticyclone decreased to $15-20 \mathrm{~cm} / \mathrm{s}$. From mid-August this gyre gradually attenuated to complete disappearance in late October. The Sukhumi anticyclone was manifested down to $300 \mathrm{~m}$ depth. Its velocity did not exceed $2-6 \mathrm{~cm} / \mathrm{s}$ there.

At the beginning of May, in the Kerch Peninsula area an anticyclonic eddy of $15-20 \mathrm{~cm} / \mathrm{s}$ velocity also occurred. By mid-June, it moved along the southern coast of Crimea to the west, to the southernmost edge of the Crimean Peninsula (Sarych Cape), increasing in diameter to about $60 \mathrm{~km}$ and shifting the Rim Current core more seaward. In early July the anticyclone decreased again and dissipated by the end of the month. It could be traced down to $400 \mathrm{~m}$ depth, where its velocity did not exceed $5-6 \mathrm{~cm} / \mathrm{s}$. In experiment 1 such formation was not found.
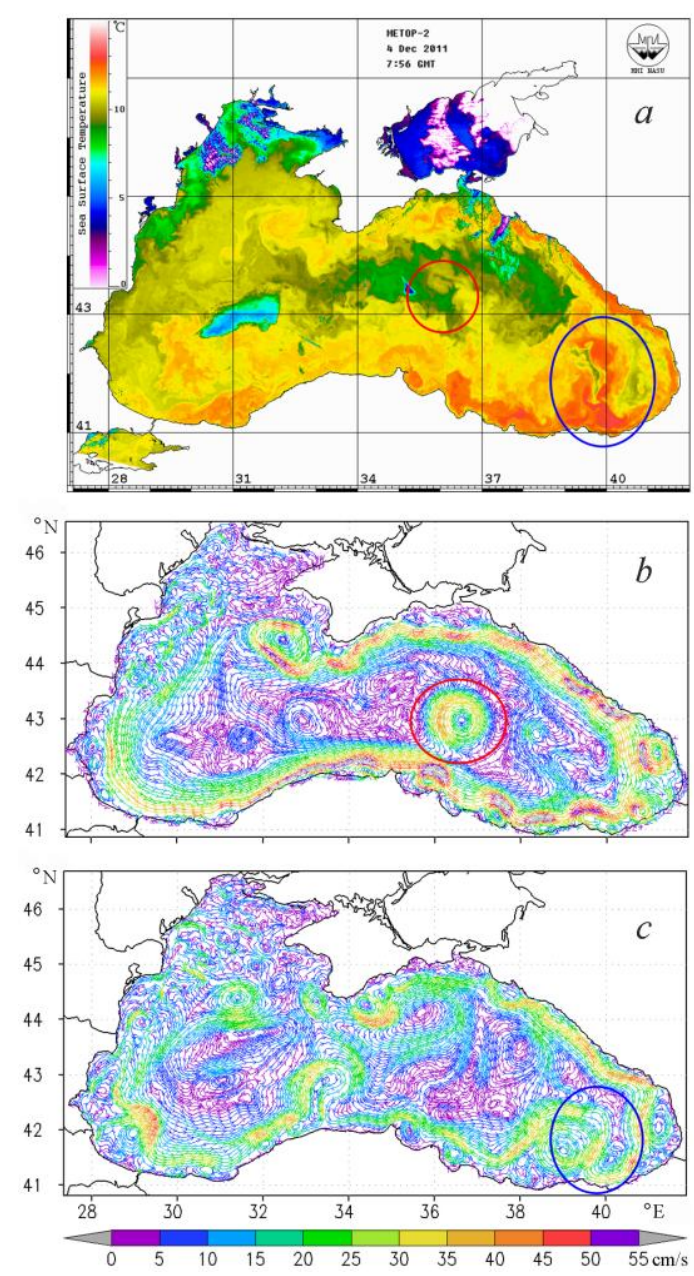

F i g. 2. SST fields based on the MetOp-2 satellite data (http://dvs.net.ru/mp/data/201112bs_sst_ru.shtml) $(a)$ and the current velocities at the $5 \mathrm{~m}$ horizon based on experiments $1(b)$ and $2(c)$ results on 04.12.2011 
The maps of the fields of reconstructed currents at $5 \mathrm{~m}$ horizon were compared with the SST images obtained from MetOp-2 satellite. An example of such a comparison for the winter season is presented in Fig. 2. A cartographic analysis of the current velocity fields and SST satellite images showed that the circulation in the Black Sea was reproduced in experiment 2 more accurately. Thus, in the satellite images (Fig. 2,a), an increased temperature zone was not observed (it characterizes the anticyclonic rotation of the waters) in that point in the sea eastern part, where in experiment $l$ (red curve in Fig. 2,b) an intense anticyclone, which was absent in the data of experiment 2, was located. Batumi anticyclone in winter period was reproduced more precisely in experiment 2 , which demonstrates the zone outlined by blue curve in Fig. 2, $a$, $c$, while in experiment $l$ in winter it was practically absent (Fig. 2, $b$ ).

According to satellite data, Batumi anticyclone consisted of three eddies of a different vorticity sign. Moreover, according to the results of experiment 1 two eddies were observed - anticyclonic and cyclonic, whereas according to experiment 2 the anticyclonic rotation of waters covered the entire southeastern part. It should be noted that the localization of Batumi anticyclone itself and the orientation of the main axes of the water rotation ellipse in experiment 2 are closer to satellite images than in experiment 1 . The structure of Sevastopol anticyclone was consistent with satellite observations according to the results of both calculations.

\section{Estimation of energy parameters}

As was shown above, when using higher resolution bathymetry data, the pattern of circulation at $10-100 \mathrm{~km}$ scales qualitatively changed, which corresponds to mesoscale classification processes [17]. Now we consider how the energy of currents and eddies in the basin changes in this case. Further in the text generally accepted English abbreviations will be used to designate the types of energy.

Basin circulation in the Black Sea is associated with mean kinetic energy $(M K E)$, and mesoscale variability is associated with eddy kinetic energy $(E K E)$. Total kinetic energy $(K E)$ is added of the mentioned components. It can also be calculated by the formula $E=\left(u^{2}+v^{2}\right) / \rho_{0}$. Having the fields of temperature, salinity, zonal and meridional velocity components for each day, we calculated their average values for the year, as well as fluctuations (deviations from the average for every day of the year) of the velocity and density fields. Based on the obtained data, for quantitative estimates of changes in the Black Sea energy over time, EKE value integrated over the volume was calculated by formula (4) from [18]. We also examined how the volume integral of the total $K E$ changed over time.

$E K E$ sources are the fluctuations of wind stresses, the transition of kinetic energy from the middle current due to velocity shift and the transition of potential energy due to available potential energy $(E P E)$. In accordance with [18], we denote the conversion rate between $E K E$ and $M K E$ as $C(E K E, M K E)$, between $E K E$ and $E P E$ - as $C(E P E, E K E)$. Energy transformation from $M K E$ to $E K E$ is due to the processes of baroclinic instability, and the energy transfer from $E P E$ to $E K E$ is due to the processes of baroclinic instability. The volume-integrated 
quantities $C(E K E, M K E)$ and $C(E P E, E K E)$ were calculated using formulas (15), (17) from [18].

In Fig. 3 the time variation of the values of volume integrals $K E$ and $E K E$ for two experiments are shown. The comparison of the curves in Fig. 3, $a$ showed that during the entire integration time $K E_{1}$ exceeded $K E_{2}$. It can be seen that the curves are qualitatively similar and close in magnitude in winter months, then since spring the difference between $K E_{1}$ and $K E_{2}$ increases, and the greatest differences are observed after October 19: $K E_{2}$ decreased by about $35 \%$ from mid-October to late November. In the warm season from May to September, a tendency toward a decrease in $K E$ was observed in both experiments, which is associated with a wind effect weakening. Wind field analysis showed that sharp increase in $K E_{1}$ and $K E_{2}$ in late October was due to the effect of a storm wind over the southwestern part of the sea. From Fig. 3, $a$ it can be seen that $K E_{2}$ almost returned to $(6-7) \cdot 10^{14} \mathrm{~J}$ level after the passage of the storm, which is close to the warm season. At the same time, $K E_{l}$ exceeded summer values until the end of the integration period.

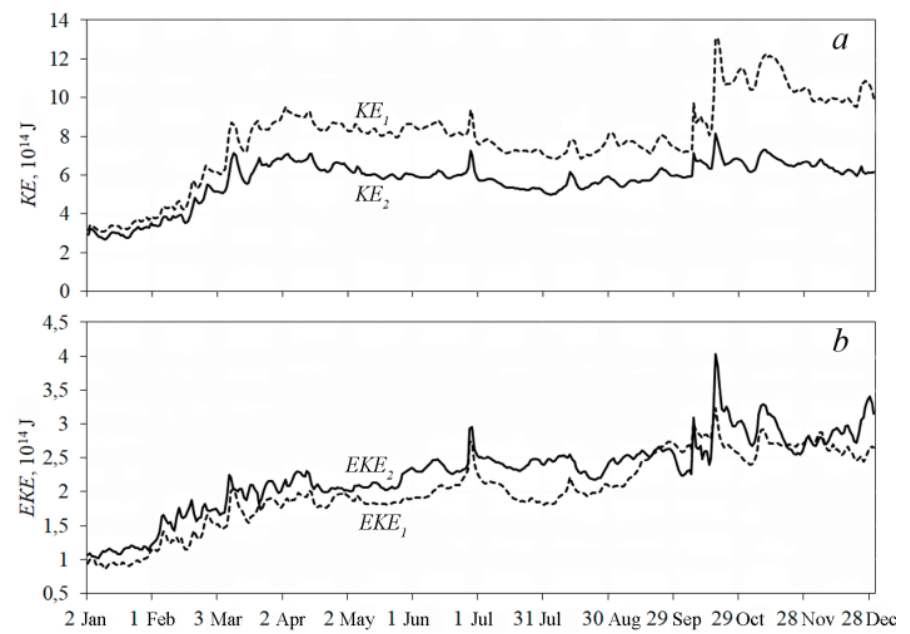

F i g. 3. Temporal variation of the volume-integrated $K E(a)$ and $E K E(b)$ in experiment 1 (hatch line) and in experiment 2 (solid line)

Eddy energy (Fig. 3, b) increased during the year in both experiments. At the same time, $E K E_{2}$ exceeded $E K E_{1}$ by, on average, 12\%. The maximum difference between $E K E_{1}$ and $E K E_{2}$ was observed on October 18, 2011. Thus, in an experiment with a smoother bottom, an extreme storm of October 18-19, 2011 led to subsequent intensification of general circulation and an increase in $K E_{l}$, while $E K E_{1}$ was a quarter of this value. In experiment $2, K E_{2}$ value increased insignificantly, while $E K E_{2}$ was about $47 \%$ of $K E_{2}$.

In order to assess what mechanisms caused such a redistribution of energy between the current and eddies, we consider the temporal variation of $C(E K E$, $M K E)$ and $C(E P E, E K E)$ integrals. In Fig 4 the curves calculated from the data of both experiments are given. During January - April, the behavior of the curves corresponding to the results of the first and second experiments was almost 
identical: the fluxes were close in value and their phases coincided. In June September, $C(E K E, M K E)_{l}$ is predominantly negative, which indicates the energy transfer from the middle current to eddies. In October, a sharp increase in $C(E K E$, $M K E)_{l}$, which led to an increase in $K E_{l}$ (dashed line in Fig. 3,a), was observed. $C(E K E, M K E)_{2}$ value in the same period made up approximately $1 \mathrm{TW}\left(10^{12} \mathrm{~W}\right)$, while $C(E K E, M K E)_{1}$ reached $6.26 \mathrm{TW}$. Significant difference in $C(E P E, E K E)$ flux magnitude is reflected by two peaks of the solid curve (experiment 2) in Fig. $4, b$ in October and November. The maximum $C(E P E, E K E)_{2}$ value was more than 4 times higher than the one of $C(E P E, E K E)_{1}$ on October 18, 2011. A comparison of the maximum values of integrals for each experiment showed that $C(E P E, E K E)_{1}$ was 3.5 times more than $C(E P E, E K E)_{1}$, and $C(E K E, M K E)_{2}$ is 7.3 times smaller than $C(E P E, E K E)_{2}$. Thus, $E C E$ growth at the end of 2011 in experiment 1 was due to the flux from the midstream to eddies, in experiment $2-$ due to the available potential energy.

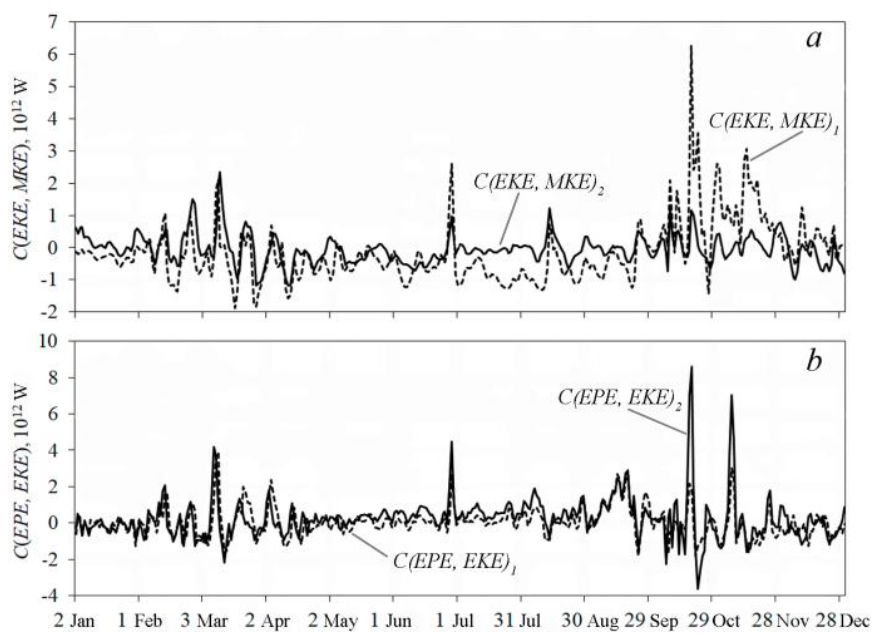

F i g. 4. Temporal variation of the volume-integrated $C(E K E, M K E)(a)$ and $C(E P E, E K E)(b)$ in experiment 1 (hatch line) and in experiment 2 (solid line)

\section{Conclusion}

A comparative analysis of the modeling results of two numerical experiments on the Black Sea circulation in 2011 using the bottom topography obtained according to MHI ODB and EMODnet is carried out. The fields of temperature, salinity, current velocity and energy characteristics calculated for each day of the studied period are considered. The accuracy of the simulation results was estimated by comparison with the data of contact measurements obtained by Argo buoys and with SST satellite images. The analysis showed that in experiment 2, at the depths where the maximum residuals between the measurements and modeling data were observed (5-30 m layer for temperature and 30-100 m for salinity), RMS of residuals decreased. At the same time, at higher horizons RMS of residuals of temperature in 0-5 m layer and salinity in 5-30 m layer increased. In 100-300 m layer RMS of residuals of both parameters decreased. At the horizons below $300 \mathrm{~m}$ the data of experiments 1 and 2 insignificantly differ from each other. 
A cartographic analysis of the reconstructed current fields and SST satellite images showed that the data from experiment 2 were more consistent with field observations. Thus, a comparison of hydrophysical fields calculated in two experiments with real data showed that the use of higher resolution bottom topography of the Black Sea provided the accuracy improvement of simulating circulation features.

The mechanisms of circulation structure variability were studied by the method of comparative analysis of the integral flux values characterizing the eddy energy and the circulation total kinetic energy, as well as the rate of energy conversion from one type to another. Temporal variation analysis of $C(E K E, M K E)$ and $C(E P E, E K E)$ integrals indicates that when using the bottom topography with a higher resolution in the modeling system, the mechanisms of energy redistribution between the currents and eddies during intense storms changed. At smoother bottom topography an increase in the kinetic energy of eddies and currents was related to barotropic instability processes. At complex rugged bathymetry, the eddy kinetic energy increased mainly due to processes associated with baroclinic instability.

\section{REFERENCES}

1. Korotaev, G.K., Sarkisyan, A.S., Knysh, V.V. and Lishaev, P.N., 2016. Reanalysis of Seasonal and Interannual Variability of Black Sea Fields for 1993-2012. Izvestiya, Atmospheric and Oceanic Physics, [e-journal] 52(4), pp. 418-430. doi:10.1134/S0001433816040071

2. Dorofeev, V.L. and Sukhikh, L.I., 2017. Modeling of Long-Term Evolution of Hydrophysical Fields of the Black Sea. Oceanology, [e-journal] 57(6), pp. 784-796. doi:10.1134/S0001437017060017

3. Demyshev, S.G., 2012. A Numerical Model of Online Forecasting Black Sea Currents. Izvestiya, Atmospheric and Oceanic Physics, [e-journal] 48(1), pp. 120-132. doi:10.1134/S0001433812010021

4. Zhuk, E., Khaliulin, A., Zodiatis, G., Nikolaidis, A., Isaeva E., 2016. Black Sea GIS developed in MHI. In: Proceedings of SPIE 9688, Fourth International Conference on Remote Sensing and Geoinformation of the Environment (RSCy2016). Cyprus, 4-8 April, 2016. 96881C. doi:10.1117/12.2241631

5. Demyshev, S.G., Korotaev, G.K. and Knysh, V.V., 2004. Modeling the Seasonal Variability of the Temperature Regime of the Black Sea Active Layer. Izvestiya, Atmospheric and Oceanic Physics, 40(2), pp. 227-237.

6. Dymova, O.A., 2017. Modeling of the Meso- and Submesoscale Dynamic Processes in the Black Sea Coastal Zones. Transactions of KarRC RAS, (8), pp. 21-30. doi:10.17076/mat585 (in Russian).

7. Demyshev, S.G. and Evstigneeva, N.A., 2016. Modeling Meso- and Sub-Mesoscale Circulation Along the Eastern Crimean Coast Using Numerical Calculations. Izvestiya, Atmospheric and Oceanic Physics, [e-journal] 52(5), pp. 560-569. doi:10.1134/S0001433816050042

8. Lyubartsev, V., Lyubartseva, S., Pinardi, N., Palzov, A., Slabakova, V., Stefanova, E., Stefanova, K., Raykov, V. and Stanchev, H. [et al.], 2018. Black Sea Checkpoint: Second Data Adequacy Report. EMODnet, 163 p. Available at: http://www.emodnet.eu/sites/emodnet.eu/files/public/Checkpoints/SecondDAR_BlackSea. pdf [Accessed: 5 January 2019].

9. Dumnov, A.D., Kirsanov, A.A., Kiseleva, E.A., Lipiyaynen, K.L., Rybal'skiy, N.G., Snakin, V.V., Afanas'ev, A.N., Borisova, O.K. and Velichko, A.A. [et al.], 2007. [National Atlas of Russia. Volume 2. Nature. Ecology]. Moscow: Kartografiya, 496 p. (in Russian).

10. Mamaev, O.I., 1963. [Oceanographic Analysis in the System $\alpha-S-T-p$ ]. Moscow: MSU, 228 p. (in Russian). 
11. Ibraev, R.A., 2001. A Study of the Sensitivity of the Model of the Black Sea Current Dynamics to the Surface Boundary Conditions. Oceanology, 41(5), pp. 615-621.

12. Mellor, G.L. and Yamada T., 1982. Development of a Turbulence Closure Model for Geophysical Fluid Problems. Reviews of Geophysics, [e-journal] 20(4), pp. 851-875. doi:10.1029/RG020i004p00851

13. Al'tman, E.N. and Simonov, A.I., eds., 1991. [Hydrometeorology and Hydrochemistry of the USSR Seas. Volume 4. The Black Sea. Release 1. Hydrometeorological Conditions]. SaintPetersburg: Gidrometeoizdat, 429 p. (in Russian).

14. Arakawa, A. and Lamb, V.R., 1981. A Potential Enstrophy and Energy Conserving Scheme for the Shallow Water Equations. Monthly Weather Review, [e-journal] 109(1), pp. 18-36. doi:10.1175/1520-0493(1981)109<0018:APEAEC>2.0.CO;2

15. Demyshev, S.G., Ivanov, V.A. and Markova, N.V., 2009. Analysis of the Black-Sea Climatic Fields Below the Main Pycnocline Obtained on the Basis of Assimilation of the Archival Data on Temperature and Salinity in the Numerical Hydrodynamic Model. Physical Oceanography, [e-journal] 19(1), pp. 1-12. doi:10.1007/s11110-009-9034-x

16. Hernandez, F., Crosnier, L., Kamachi, M., Maes, C., Oke, P. and Verbrugge, N., 2006. List of Internal Metrics the MERSEA-GODAE Global Ocean: Specification for Implementation. MERSEA, 71 p. Available at: http://www.clivar.org/sites/default/files/documents/wgomd/GODAE_MERSEA-report.pdf [Accessed: 5 January 2019].

17. Chelton, D.B., 2001. Overview of the High-Resolution Ocean Topography: Science Working Group Meeting. In: D. B. Chelton, ed., 2001. Report of the High-Resolution Ocean Topography: Science Working Group Meeting. Corvallis, Oregon: Oregon State University, p. 1-19. Available at: https://citeseerx.ist.psu.edu/viewdoc/download?doi=10.1.1.582.3299\&rep=rep1\&type=pdf [Accessed: 5 January 2019].

18. von Storch, J.-S., Eden, C., Fast, I., Haak, H., Hernández-Deckers, D., Maier-Reimer, E., Marotzke, J. and Stammer, D., 2012. An Estimate of the Lorenz Energy Cycle for the World Ocean Based on the $1 / 10^{\circ}$ STORM/NCEP Simulation. Journal of Physical Oceanography, [e-journal] 42(12), pp. 2185-2205. doi:10.1175/JPO-D-12-079.1

About the authors:

Ol'ga A. Dymova - Senior research Associate, Marine Hydrophysical Institute of RAS (2 Kapitanskaya Str., Sevastopol, 299011, Russian Federation), Ph.D. (Phys.-Math.), SPIN-code: 796-208, ORCID ID: 0000-0003-4036-2447, ResearcherID: P-9669-2015, olgadym@ yahoo.com

Nadezhda A. Miklashevskaya - Junior Research Associate, Marine Hydrophysical Institute of RAS (2 Kapitanskaya Str., Sevastopol, 299011, Russian Federation), ORCID ID: 0000-0003-2619-343X, nmikl@rambler.ru

Contribution of the co-authors:

Ol'ga A. Dymova - problem formulation and statement, carrying out numerical experiments, analysis and interpretation of research results, formulation of conclusions, editing and addition of the paper text

Nadezhda A. Miklashevskaya - comparison with observational data, qualitative analysis of the study results and preparation of initial conclusions, preparation of the paper text

All the authors have read and approved the final manuscript.

The authors declare that they have no conflict of interest. 